\title{
BMJ Open The clinical characteristics of patients with glaucoma presenting to Botswana healthcare facilities: an observational study
}

Daniel J Jackson, ${ }^{1}$ Mohammad S Razai, ${ }^{1}$ Rosemary Falama, ${ }^{2}$ Matlhogonolo Mongwa, ${ }^{2}$ Mishell Mutapanduwa, ${ }^{2}$ Chao Baemisi, ${ }^{2}$ Engelinah Josiah, ${ }^{2}$ Oathokwa Nkomazana, ${ }^{2}$ Alice Lehasa, ${ }^{3}$ Evelyn Brealey, ${ }^{4}$ Andrew J White, ${ }^{5}$ Deborah Jankowski, ${ }^{6}$ Malcolm G Kerr-Muir, ${ }^{6}$ Keith R Martin, ${ }^{5}$ Jeremiah M Ngondi ${ }^{7}$

To cite: Jackson DJ, Razai MS, Falama R, et al. The clinical characteristics of patients with glaucoma presenting to

Botswana healthcare facilities: an observational study. $B M J$ Open 2014;4:e005965. doi:10.1136/bmjopen-2014005965

- Prepublication history for this paper is available online. To view these files please visit the journal online (http://dx.doi.org/10.1136/ bmjopen-2014-005965)

Received 24 June 2014 Accepted 19 November 2014

\section{CrossMark}

For numbered affiliations see end of article.

Correspondence to Dr Daniel J Jackson; daniel.jackson@doctors.org. uk

\section{ABSTRACT}

Objective: This study aimed to establish the clinical characteristics of patients with glaucoma attending eye care facilities in Botswana, and management of glaucoma among patients who received care in these facilities.

The study also aimed to calculate the number of new diagnoses of glaucoma within the glaucoma service.

Design: A prospective, hospital-based, observational study.

Setting: A multicentre study was undertaken in government-run eye departments in Botswana from June to August 2012.

Participants: All patients with a diagnosis of glaucoma attending clinics at seven study sites were invited to participate.

Outcome measures: Examination findings, diagnosis and management were extracted from individual patientheld medical charts. Sociodemographic characteristics, patient knowledge and understanding of glaucoma were assessed through face-to-face interviews. In addition, details of outpatient attendances for 2011 were collected from 21 government-run hospitals.

Results: The majority of the 366 patients interviewed had a diagnosis of primary glaucoma (86.6\%). The diagnoses were mainly made by ophthalmologists $(48.6 \%)$ and ophthalmic nurses (44.0\%). Many patients $(38.5 \%)$ had been symptomatic for over 6 months before visiting an eye clinic. The mean presenting intraocular pressure was $28.2 \mathrm{~mm} \mathrm{Hg}$ (SD $11.9 \mathrm{~mm} \mathrm{Hg}$ ). Most follow-up patients $(79.2 \%)$ had not received surgery, however, many $(89.5 \%)$ would accept surgery. Only $11.5 \%$ of participants had heard of glaucoma prior to diagnosis. Many participants (35.9\%) did not understand glaucoma after being diagnosed. The majority $(94.9 \%)$ of living first-degree relatives had never been examined. The number of newly diagnosed glaucoma cases for 2011 in the south of the country was $14.1 / 100000 ; 95 \% \mathrm{Cl}$ (12.0 to 16.5), in the north it was $16.2 / 100000 ; 95 \% \mathrm{Cl}$ (13.8 to 19.0).

Conclusions: Glaucoma is a significant burden that presents challenges to ophthalmic services in Botswana. Many patients have limited understanding of the

\section{Strengths and limitations of this study}

- The study recruited a large number of patients from a sparsely populated country where the number of patients with glaucoma being managed by the eye service is unknown.

- This study provided detailed demographic and clinical data from a previously uninvestigated patient population where individual patient records are not kept.

- The weakness of the study is that it is hospital based rather than population based, which will be limited in reflecting the true population when guiding service development.

- Limited availability of sensitive diagnostic equipment and the reliance on ophthalmic nurses will lead to inaccuracy in the diagnosis and classification of glaucoma.

- The reliance on often incomplete medical records and collection of historical information from patient interview will introduce error in the data collected.

condition and poor access to services. There is a need to develop a treatment infrastructure to include safe surgery and a reliable supply of effective medication.

\section{BACKGROUND}

Glaucoma is a significant cause of visual impairment, estimated to be responsible for $8 \%$ of blindness throughout the world. ${ }^{1}$ It is also a major cause of blindness in Africa, ${ }^{2-5}$ with black populations having the highest prevalence of primary open-angle glaucoma (POAG). ${ }^{6-8}$

Studies in other sub-Saharan countries have shown that POAG is the most common form of glaucoma. ${ }^{2-5}$ The disease tends to 
have an earlier onset, and the progression rate may also be faster in these populations. ${ }^{9} 10$

There are significant challenges with diagnosis and management of glaucoma in the developing world. Many patients have poor access to healthcare facilities and receive inadequate follow-up care. ${ }^{11-18}$ The problem is considerably aggravated by poor awareness of glaucoma among the population leading to late presentation, ${ }^{19-25}$ and limited treatment options once they are diagnosed with the disease.

Botswana is a middle-income African country with an estimated population of 2.24 million. ${ }^{26}$ There is limited access to ophthalmic care, with only two ophthalmologists working in the public sector during the data collection period. There have been no previous studies investigating glaucoma in Botswana and very little information is available regarding glaucoma burden and its management strategies in the country.

This study, first, aimed to investigate the characteristics of patients with glaucoma in Botswana through describing the type of glaucoma and presenting symptoms; determining how glaucoma is managed in these patients; establishing prior awareness of glaucoma; and exploring understanding of glaucoma after diagnosis. Second, the study attempted to estimate the number of new diagnoses of glaucoma within these eye facilities for 2011 .

\section{METHODS}

\section{Data collection}

Patient interviews were conducted by the principal investigators in seven government-run institutions over a 7 -week period (18 June-3 August 2012). The institutions consisted of the two tertiary referral centres: Princess Marina Hospital (PMH) and Sekgoma Memorial Hospital (SMH); a referral hospital: Nyangabgwe; district hospitals: Kanye, Mahalapye, Palapye; and primary hospitals: Letlhakane, Palapye. Study site selection was based on which eye departments see the most patients.

At the time of the study, there were two ophthalmologists working in the government sector, one based at each tertiary referral centre. The referral centre for the south is PMH; the one for the north is SMH. The majority of eye clinics, including the two tertiary centres, did not keep registers of the number of patients with glaucoma. In addition, there were no records of referral sources in either of the tertiary hospitals. Hospitals kept records of the number of consultations by diagnosis and whether a consultation was a new or repeat visit. Individual patient records were not kept at the hospitals. Instead, patients brought a card detailing diagnosis, examination findings and treatment when attending an appointment. These patient-held cards are taken to outpatient clinic appointments at all hospitals and are the only record of the details of a consultation.

A drop-in service was organised by every government eye clinic, whereby patients attended to be treated within the eye clinic or referred to either $\mathrm{PMH}$ or $\mathrm{SMH}$ to see an ophthalmologist. There were no referral guidelines and there was no defined referral pathway.

All patients with a diagnosis of glaucoma attending an outpatient clinic at one of the study sites were invited to participate and were interviewed using a structured questionnaire. There was no active recruitment or advertising. The questionnaire was designed by the authors of the study and translated into Setswana and subsequently backtranslated. It was then piloted on 12 patients attending clinic at PMH prior to data collection and adjustments were made on the basis of the response obtained. Interviews were conducted in English or Setswana with the help of an interpreter. Diagnosis of glaucoma was made through patient history and examination. The examination of patients varied between clinics, depending on equipment available and expertise of ophthalmic staff. All patients who participated in the survey had their diagnosis of glaucoma confirmed by an ophthalmologist at a point after their diagnosis. Data collected in these interviews included sociodemographic characteristics, history, family history, diagnosis, treatment, prior awareness of glaucoma, information received after diagnosis and referral sources. Information on presenting complaint and duration of presenting complaint at first presentation that led to the diagnosis of glaucoma was collected from patient held records if it had been recorded, or collected from the patient if not recorded in the patient file. Past medical history was assessed by inspecting the patient record file for confirmed diagnoses of hypertension, diabetes, myopia, eye injury, previous trauma, previous eye surgery, migraine and steroid use. The patients were also asked if they knew that they suffered from such problems. Family history was assessed by checking medical cards for any details and asking if any member of that patient's family was diagnosed with glaucoma or if any member of that patient's family was blind. Current understanding of glaucoma was assessed by asking patients what they understood of glaucoma. A patient was deemed to understand glaucoma if they gave answers such as "glaucoma is high pressure in the eye/damages the nerve/is an eye condition that could lead to blindness/may need treatment for life." Screening of relatives was assessed by asking patients if any of their family members had ever been checked for glaucoma or if any of their family members had ever been to an eye clinic. Information on examination findings, management and history were obtained from patients' medical cards.

In the second aspect of the study, the total number of new and follow-up glaucoma visits was collected from clinic logbooks in 21 centres for each month of 2011 to estimate the number of newly diagnosed patients within the glaucoma service for that year. The study sites consisted of the two eye referral centres with the remaining sites being primary and district hospitals. The selection criteria for the government hospitals were any institution with a functional eye unit as determined by the Ministry of Health. At the time of study, there were 27 government eye units; 6 declined to participate. 


\section{Data management and analysis}

Data were entered in Microsoft Excel worksheets and analysis undertaken using Stata V.9.2 (Stata Corporation, College Station, Texas, USA). Data were explored using cross-tabulations and frequency distributions. Visual acuity was classified based on the WHO categories of visual impairment. Severe impairment was defined as visual acuity of less than $6 / 60$ but greater than or equal to $3 / 60$. Moderate impairment was defined as visual acuity of less than $6 / 18$ but equal to or greater than $6 / 60$ in the better eye. Normal vision was defined to represent persons who had normal or near-normal vision in the better eye (VA $\geq 6 / 18)$. Blindness or visual impairment due to field restriction was not included due to lack of regular visual field testing. The number of newly diagnosed cases of glaucoma in Botswana in 2011 per unit population was calculated for $\mathrm{PMH}$ as well as SMH by dividing the number of patients with newly diagnosed glaucoma by the catchment population served by each referral hospital. CIs were estimated using binomial exact method. An assumption was made that most district and primary hospitals refer patient with newly diagnosed glaucoma to either SMH or PMH. Written informed consent was sought and obtained from all patients participating in the study.

\section{RESULTS}

\section{Demographic characteristics}

A total of 366 patients participated in the glaucoma survey. Ninety-eight per cent of patients participated. The demographic characteristics of the glaucoma survey participants are summarised in table 1 . The majority of the patients were female (52.5\%), aged 60 years and above $(62.3 \%)$ and $24.2 \%$ had no formal education. The mean age was 62 years (SD 17.2 years).

\section{Presenting symptoms, duration and medical history}

Table 2 summarises the presenting symptoms and its history among glaucoma survey participants. The most common presenting symptom at first presentation was poor vision $(66.4 \%)$. Many cases were detected incidentally through routine check-up. Other symptoms on presentation included red or itchy eyes, headache, pain and tearing. A significant proportion of patients (38.5\%) waited over 6 months from the beginning of their symptom before visiting an eye clinic. The majority of patients $(51.1 \%)$ had visited an eye clinic other than the one they attended when interviewed. Many $(37.1 \%)$ had a known family history and $30.3 \%$ of patients had a firstdegree relative diagnosed with glaucoma. Fourteen participants had one or more family members with significantly impaired vision of unknown cause. Based on the WHO categories of visual impairment, $13.7 \%$ of the patients interviewed were blind and $53.6 \%$ had normal or near normal vision. Blindness was defined as a visual acuity of less than 3/60 in the better eye. Many (22.3\%) patients had a travel time of over $1 \mathrm{~h}$ to visit an eye clinic.
Table 1 Demographic characteristics of glaucoma survey participants

\begin{tabular}{lc}
\hline Characteristic & Number of patients (\%) \\
\hline Sex & \\
Male & $174(47.5)$ \\
Female & $192(52.5)$ \\
Age & \\
$0-19$ & $14(3.8)$ \\
$20-29$ & $4(1.1)$ \\
$30-39$ & $24(6.6)$ \\
$40-49$ & $36(9.8)$ \\
$50-59$ & $58(15.8)$ \\
$60-69$ & $92(25.1)$ \\
$70-79$ & $86(23.5)$ \\
$>79$ & $50(13.7)$ \\
Unknown & $2(0.5)$ \\
Education & \\
None & $88(24.2)$ \\
Primary & $178(48.9)$ \\
Secondary & $64(17.6)$ \\
Graduate/postgraduate & $34(9.3)$ \\
Marital status & \\
Single & $125(34.4)$ \\
Married & $154(42.4)$ \\
Divorced & $9(2.5)$ \\
Widowed & $74(20.4)$ \\
Separated & $1(0.3)$ \\
\hline
\end{tabular}

Table 2 Summary of presenting symptoms, duration and medical history among patients with glaucoma

\begin{tabular}{lc}
\hline Characteristics & Number of patients (\%) \\
\hline Presenting symptom & $20(5.5)$ \\
None & $243(66.4)$ \\
Poor vision & $31(8.5)$ \\
Inability to read & $15(4.1)$ \\
Bumping into objects & $74(20.2)$ \\
Other & \\
Duration & $225(61.5)$ \\
$<6$ months & $141(38.5)$ \\
$>6$ months & \\
Medical history & $187(51.1)$ \\
Hypertension & $44(12.0)$ \\
Diabetes & $115(31.4)$ \\
Myopia & $59(16.1)$ \\
Eye injury & $114(31.1)$ \\
Previous eye surgery & $132(36.1)$ \\
Migraine & $60(16.4)$ \\
Steroid use & \\
Travel taken (h) (n=359) & $211(58.8)$ \\
Less than 0.5 & $68(18.9)$ \\
Between 0.5 and 1 & $80(22.3)$ \\
Greater than 1 & $187(51.1)$ \\
Visits to other hospitals & $128(37.1)$ \\
Number with family history & \\
Visual acuity & $196(53.6)$ \\
Normal vision & $76(20.8)$ \\
Moderate impairment & $39(10.7)$ \\
Severe impairment & $50(13.7)$ \\
Blind & \\
\hline
\end{tabular}


Diagnosis and management of glaucoma

Table 3 summarises the cadre of eye-care practitioners diagnosing glaucoma, type of glaucoma and management of the participants. The majority of initial diagnoses were made by ophthalmologists $(48.6 \%)$ and ophthalmic nurses $(44.0 \%)$. The vast majority of cases were due to primary glaucoma $(86.6 \%)$. Secondary glaucoma types included: phacomorphic, congenital, pigmentary, uveitic, neovascular and traumatic glaucoma. The most common medications used were levobunolol $(72.7 \%)$ and brimonidine (59\%). Most patients (79.2\%) did not have surgery to treat glaucoma. A minority $(6.0 \%)$ had not received either surgical or medical treatment. Every patient interviewed reported difficulty in obtaining medications from government hospitals and had to either buy medication through private facilities or wait for medications to become available.

From the patient-held medical card, presenting intraocular pressure (IOP) was recorded in 204 participants. Of those, $175(85.8 \%)$ had an IOP of greater than $21 \mathrm{~mm} \mathrm{Hg}$ in one or both eyes at first presentation. The mean presenting IOP was $28.2 \mathrm{~mm} \mathrm{Hg}$ ( $\mathrm{SD} 11.9 \mathrm{~mm} \mathrm{Hg}$ ); the median presenting IOP was $25 \mathrm{~mm} \mathrm{Hg}$. Current vertical

Table 3 Summary of diagnosis, glaucoma type and management of glaucoma

\begin{tabular}{lc}
\hline Characteristic & Number of patients (\%) \\
\hline Diagnosed by & \\
Ophthalmologist & $178(48.6)$ \\
Ophthalmic nurse & $161(44.0)$ \\
Other doctor & $13(3.6)$ \\
Optometrist & $10(2.7)$ \\
Unknown & $4(1.1)$ \\
Glaucoma type & \\
Primary & $317(86.6)$ \\
Secondary & $30(8.2)$ \\
Unknown & $19(5.19)$ \\
Anterior chamber angle & \\
Open angle & $316(86.3)$ \\
Angle closure & $2(0.5)$ \\
Unknown & $48(13.1)$ \\
Eye affected & \\
Right eye only & $21(5.7)$ \\
Left eye only & $34(9.3)$ \\
Both eyes & $287(78.4)$ \\
Unknown & $24(6.6)$ \\
Medication & \\
Brimonidine & $216(59.0)$ \\
Levobunolol & $266(72.7)$ \\
Pilocarpine & $138(37.7)$ \\
Acetazolamide & $59(16.1)$ \\
Latanoprost & $3(0.8)$ \\
Oxymetazoline & $2(0.5)$ \\
No medication & $26(7.1)$ \\
Glaucoma surgery & \\
Yes & $77(21.1)$ \\
No & $289(79.2)$ \\
No treatment & $22(6.0)$ \\
\hline
\end{tabular}

cup/disc ratio (CDR) was recorded from 144 participants; $133(92.4 \%)$ had a CDR of 0.6 or above in at least one eye.

\section{Patients' knowledge of glaucoma before and after diagnosis}

Table 4 summarises the participants' awareness and knowledge of glaucoma before diagnosis and after diagnosis. Only $11.5 \%$ of the participants had heard of glaucoma prior to being diagnosed. Many participants $(35.9 \%)$ did not understand glaucoma after being diagnosed.

\section{Screening of relatives for glaucoma}

The screening of living first-degree relatives of patients with primary glaucoma is shown in table 5 . The vast majority of living relatives $(94.9 \%)$ have never been examined for glaucoma. The highest proportion of relatives checked was parents of patients with glaucoma $(11.1 \%)$.

\section{Number of newly diagnosed cases of glaucoma}

The number of new glaucoma cases at PMH in 2011 was 157. PMH serves a catchment population of 1114589 ; therefore the number of newly diagnosed cases of glaucoma in the south of the country was 14.1/100 000; $95 \%$ CI (12.0 to 16.5). The number of new glaucoma cases at SMH in 2011 was 154. SMH serves a population of 949312 , thus the number of newly diagnosed cases of glaucoma in the north of the country is $16.2 / 100000$; 95\% CI (13.8 to 19.0$)$. There was no statistically significant difference in the number of new cases diagnosed in the south compared to the north ( $p$ value $=0.2$ ).

Table 4 Summary of patients' knowledge of glaucoma before and after diagnosis

\begin{tabular}{ll}
\hline Knowledge & $\begin{array}{l}\text { Number of } \\
\text { patients (\%) }\end{array}$
\end{tabular}

Before diagnosis

Number who had heard of glaucoma

$(\mathrm{N}=366)$

Of those who have heard of glaucoma $(\mathrm{N}=42)$

Number aware that it can cause vision loss

Number who believed vision loss from glaucoma can be reversed Number aware it could run in families Number aware of the need for lifelong treatment

Number who would accept an offer of surgery $(\mathrm{N}=366)$

Number who have taken traditional medication for their eyes $(\mathrm{N}=366)$

After diagnosis

Number who understand glaucoma $(\mathrm{N}=366)$

Of those who understood $(\mathrm{N}=229)$

Number briefed

Number who had received leaflet 
Table 5 Examination of living relatives of patients with primary glaucoma

\begin{tabular}{lll}
\hline Relation & Total living relatives & Screened (\%) \\
\hline Brothers & 449 & $26(5.8)$ \\
Sisters & 491 & $23(4.7)$ \\
Parents & 108 & $12(11.1)$ \\
Sons & 638 & $28(4.4)$ \\
Daughters & 638 & $29(4.5)$ \\
\hline
\end{tabular}

\section{DISCUSSION}

This study aimed to explore the characteristics of patients with glaucoma and how these patients were managed by the eye services in Botswana. The study revealed that: the majority of patients had primary glaucoma; diagnoses were mainly made by ophthalmologists and ophthalmic nurses; the majority of patients did not undergo surgical treatment; few patients understood glaucoma before and after diagnosis; and the majority of living first-degree relatives had never been screened for glaucoma. The number of newly diagnosed cases of glaucoma in the south of the country was 14.1/100 000; $95 \%$ CI (12.0 to 16.5$)$, in the north it was $16.2 / 100000$; 95\% CI (13.8 to 19.0$)$.

There are several limitations of the study. A hospitalbased as opposed to a population-based study is subject to bias as attendance at such clinics is dependent on several variables such as education, transport, wealth, previous experience and family history. Therefore, the sample is not representative of the whole population as only the patients who sought help were interviewed. The absence of comprehensive hospital records caused difficulty in estimating numbers of patients with glaucoma and has resulted in several challenges to the eye-care services within the country. Additionally, the records brought to the clinics by individual patients were often incomplete and patients could not always give a clear description of events. Certain examination findings, for example, CDR, were not being recorded by ophthalmic staff in the patient-held notes. Such historical data collected from patient interviews can be inaccurate, potentially leading to errors in presenting symptom, duration, medical history and family history. The introduction of standardised patient files to the eye services of Botswana may encourage consistent completion of important details during an eye consultation. Over half $(51.1 \%)$ of patients had visited more than one hospital for their eye conditions, therefore incomplete glaucoma registers and lack of referral records may make follow-up particularly difficult as patients become 'lost' in the system.

Six units did not participate; these were small primary hospitals that we have been told to see a small number of patients with glaucoma compared with the clinics included in the study. We understand that most of their patients with glaucoma are referred to tertiary centres, therefore we anticipate the missing data from these small centres had a small effect on the results and on our calculation of the number of new diagnoses within the glaucoma service.

Many patients were unaware of glaucoma before diagnosis, and those who did had a limited understanding of the disease, which is similar to findings in other African countries. ${ }^{27-34}$ Indeed, $66.4 \%$ of patients had poor vision as their presenting symptom and $38.5 \%$ of patients were symptomatic for over 6 months before seeking help, suggesting that many of these patients are presenting late and only after significant visual loss has occurred. Public awareness campaigns could be used to highlight glaucoma as a significant and silent cause of blindness. Previous studies have identified a number of successful ways to deliver and communicate effective health education, mainly through media outlets such as radio and television. ${ }^{35}$ The disadvantages of such programmes would be the implementation, cost and the need to divide such resources for other health problems of considerable importance in the country, where awareness is limited. A large proportion of patients diagnosed did not understand the disease and the aims of medical therapy after diagnosis, which leads to incorrect administration of medication and poor compliance. Basic education schemes have been used elsewhere to increase patients' knowledge and such programmes may also manage expectations of glaucoma therapy. ${ }^{36}$ Such schemes could be used in Botswana to assist in teaching correct administration of eye drops and improving compliance with medical therapy.

Large numbers of at risk family members were not checked for primary glaucoma, therefore large numbers of people in this at-risk group may unknowingly have the disease. The reasons for this include lack of awareness of the disease and poor access to ophthalmic services. Community eye outreach programmes have had success in detecting glaucoma in earlier stages in some African countries, ${ }^{37}$ although screening programmes are still of unproven benefit. Botswana is a sparsely populated country, therefore, any future service development must include a robust outreach scheme to remote areas.

There is limited availability of medications and almost complete lack of prostaglandin drugs. A large proportion $(21.1 \%)$ of the patients interviewed had received glaucoma surgery. The vast majority were trabeculectomy; many were unaugmented as antimetabolites were only available at PMH and were frequently out of stock. Of the 77 patients who had surgery, 70 were using topical medication suggesting a suboptimal outcome. In 2011, a total of 3099 ophthalmic operations were undertaken across six government-run hospitals. Only $0.8 \%$ of these were glaucoma operations. The large proportion of patients having had glaucoma surgery in our study could be due to this subgroup of patients being more proactive and therefore more likely to attend follow-up or having better access to eye clinics. Alternatively, some patients had seen private ophthalmologists in Botswana or abroad, mainly in South Africa. There were four ophthalmologists working in the private sector, three in Gaborone and one in Francistown. 
Surgery as a first-line intervention has been advocated in other African countries. ${ }^{11} 38$ However, it is believed that patients are likely to refuse ocular surgery due to rumours of failed surgeries within small communities ${ }^{39}$ and because glaucoma surgery is associated with appreciable risk and will not improve vision. Interestingly, the majority of patients interviewed in Botswana would consider surgery to treat their glaucoma, which if they went through with it, is a higher acceptance rate than has been found in some other studies. ${ }^{40} \mathrm{~A}$ safe and cost-effective treatment where the majority of follow-ups could be performed by mid-level eye-care workers would be ideal. Selective Laser Trabeculoplasty (SLT) has been advocated as a primary treatment for open-angle glaucoma ${ }^{41}$ and there is evidence that shows efficacy of SLT as either an adjunct or an alternative to medical therapy where access to medications or compliance is a problem. ${ }^{42}$

The majority of patients had a diagnosis of primary glaucoma, which has been shown to be the main type of glaucoma in African populations. ${ }^{2-5}$ However, the number of angle closure glaucoma diagnoses made by the ophthalmic staff may be an underestimate due to lack of gonioscopic lenses in certain eye clinics. At the time of the study, functioning goniscopic lenses were only present in PMH and Nyangabgwe. This is of concern as angle closure has been shown to have a prevalence of $0.5-1.0 \%$ in similar populations. ${ }^{4} 43$ A large proportion $(44 \%)$ of patients with glaucoma in the study were originally diagnosed by ophthalmic nurses. While these patients had been referred to an ophthalmologist to have the diagnosis verified, there are some clinics that do not routinely refer all patients with glaucoma/glaucoma-suspect to an ophthalmologist. In addition, only three clinics had visual field analysers and five clinics were without tonometers. IOP was usually measured using Schiotz or non-contact air puff tonometers; one clinic had a Goldmann applanation tonometer. The lack of sensitive diagnostic equipment and reliance on unsupported ophthalmic nurses to make initial diagnoses will likely mean a proportion of patients in the glaucoma service have been misdiagnosed.

There is a lack of reliable epidemiological data on the incidence and prevalence of glaucoma in Africa. The prevalence in Botswana is unlikely to be very different from the two population-based studies in Tswana populations of South Africa of similar ethnicity. The adjusted prevalence was $2.9 \%^{4}{ }^{43}$ for POAG and $2 \%$ for secondary glaucoma. ${ }^{4}$ Interestingly, a diagnosis of secondary glaucoma was given to only $8.2 \%$ of the patients in our sample, suggesting that it may be unrecognised or misdiagnosed as primary glaucoma.

The number of newly diagnosed glaucoma cases in Botswana is likely to be an underestimate of the incidence. This is partly because some peripheral clinics did not refer all patients with newly diagnosed glaucoma or glaucoma suspect to the referral centres. There will also be a number of patients referred who do not attend appointments at either tertiary centre. Glaucoma is usually asymptomatic in the early disease stages and therefore this study will miss these patients who will not present to eye clinics. Many family members of patients with glaucoma had not been checked, raising the possibility that the true burden of glaucoma is much higher than estimated in Botswana, with many patients unknown to the eye care services. Indeed, conservative estimates of the annual incidence of glaucoma in other African countries have been higher at approximately 400 cases per 1 million of the population. ${ }^{11}$ This suggests the true annual incidence of glaucoma could be double what is being diagnosed by the glaucoma services in Botswana.

Conclusions from this study must be interpreted cautiously. Glaucoma is a challenge to the eye services in the country, as some patients have poor access to services, limited awareness and understanding of the disease, and limited treatment options from the resources available. Further research into the incidence and prevalence of glaucoma in Botswana is required, as well as the need to establish effective treatments in eye health service.

Author affiliations

${ }^{1}$ University of Cambridge School of Clinical Medicine, Addenbrooke's Hospital, Cambridge, UK

${ }^{2}$ University of Botswana School of Medicine, Gaborone, Botswana

${ }^{3}$ Ministry of Health, Gaborone, Botswana

${ }^{4}$ Addenbrooke's Abroad, Addenbrooke's Hospital, Cambridge, UK

${ }^{5}$ Cambridge NIHR Biomedical Research Centre, University of Cambridge, Cambridge, UK

${ }^{6}$ Department of Ophthalmology, Addenbrooke's Hospital, Cambridge, UK

${ }^{7}$ Institute of Public Health, University of Cambridge, Cambridge, UK

Acknowledgements The authors are grateful to Addenbrooke's Abroad, Addenbrooke's Charitable Trust, for facilitating this study and providing support.

Contributors All authors contributed to the design and implementation of the study; and drafted and provided approval of the final manuscript. DJJ, MSR, $\mathrm{RF}, \mathrm{MMo}, \mathrm{MMu}, \mathrm{EJ}$ and $\mathrm{CB}$ were responsible for data collection.

Funding This research received no specific grant from any funding agency in the public, commercial or not-for-profit sectors.

\section{Competing interests None.}

Ethics approval Institutional Review Board approval and ethical clearance were obtained from the Ministry of Health of the Republic of Botswana and the relevant health facilities.

Provenance and peer review Not commissioned; externally peer reviewed.

Data sharing statement No additional data are available.

Open Access This is an Open Access article distributed in accordance with the Creative Commons Attribution Non Commercial (CC BY-NC 4.0) license, which permits others to distribute, remix, adapt, build upon this work noncommercially, and license their derivative works on different terms, provided the original work is properly cited and the use is non-commercial. See: http:// creativecommons.org/licenses/by-nc/4.0/

\section{REFERENCES}

1. Pascolini D, Mariotti SP. Global estimates of visual impairment: 2010. Br J Ophthalmol 2012;96:614-18.

2. Buhrmann RR, Quigley HA, Barron $Y$, et al. Prevalence of glaucoma in a rural East African population. Invest Ophthalmol Vis Sci 2000;41:40-8.

3. Rotchford AP, Johnson GJ. Glaucoma in Zulus. A population-based cross-sectional survey in a rural district in South Africa. Arch Ophthalmol 2002;120:471-8. 
4. Rotchford AP, Kirwan JF, Muller MA, et al. Temba glaucoma study: a population-based cross-sectional survey in urban South Africa. Ophthalmology 2003;110:376-82.

5. Ntim-Amponsah CT, Amoaku WM, Ofosu-Amaah S, et al. Prevalence of glaucoma in an African population. Eye (Lond) 2004;18:491-7.

6. Racette L, Wilson MR, Zangwill LM, et al. Primary open-angle glaucoma in blacks: a review. Surv Ophthalmol 2003;48:295-313.

7. Cook C, Foster P. Epidemiology of glaucoma: what's new? Can $J$ Ophthalmol 2012;47:223-6.

8. Rudnicka AR, Mt-Isa S, Owen CG, et al. Variations in primary open-angle glaucoma prevalence by age, gender, and race: a Bayesian meta-analysis. Invest Ophthalmol Vis Sci 2006;47:4254-61.

9. Omoti AE. Glaucoma in Benin City, Nigeria. Niger Postgrad Med J 2005;12:189-92.

10. Majekodunmi S. Glaucoma in Lagos. Ghana Med J 1978;17:23-6.

11. Cook C. Glaucoma in Africa: size of the problem and possible solutions. J Glaucoma 2009;18:124-8.

12. Egbert PR. Glaucoma in West Africa: a neglected problem. Br J Ophthalmol 2002;86:131-2.

13. Chuka-Okosa CM, Faal HB. Glaucoma services in the Gambia. Niger J Ophthalmol 2003;11:19-23.

14. Omoti AE. A review of the choice of therapy in primary open angle glaucoma. Niger J Clin Pract 2005;8:29-34.

15. Rotchford $A$. What is practical in glaucoma management? Eye (Lond) 2005;19:1125-32.

16. Olatunji FO, Ibrahim UF, Muhammad N, et al. Challenges of glaucoma service delivery in Federal Medical Centre, Azare, Nigeria. Afr J Med Med Sci 2008;37:355-9.

17. Standefer JE. Challenges in glaucoma management in developing countries: Is vision 2020 ready for glaucoma? Niger J Ophthalmol 2009;18:1-2.

18. Patel D, Mercer E, Mason I. Ophthalmic equipment survey 2010: preliminary results. Community Eye Health 2010;23:22-5.

19. Ashaye AO. Clinical features of primary glaucoma in Ibadan. Niger $J$ Ophthalmol 2003;11:70-5.

20. Lawan A. Pattern of presentation and outcome of surgical management of primary open angle glaucoma in Kano, Northern Nigeria. Ann Afr Med 2007;6:180-5.

21. Gyasi M, Amoako W, Adjuik M. Presentation patterns of primary open angle glaucomas in North Eastern Ghana. Ghana Med $J$ 2010;44:25-30.

22. Chukwuka IO, Ejimadu CS, Pedro-Egbe CN. Clinical features of primary glaucoma in South East Nigeria. Ann Biomed Sci 2012;11:88-95.

23. Omoti $\mathrm{AE}$, Osahon Al, Waziri-Erameh MJ. Pattern of presentation of primary open-angle glaucoma in Benin City, Nigeria. Trop Doct 2006;36:97-100.

24. Olatunji FO, Ibrahim UF, Muhammad N, et al. The types and treatment of glaucoma among adults in North Eastern part of Nigeria. Tanzan Med J 2009;24:24-8.

25. Faal H. Primary open-angle glaucoma: everyone's business. Community Eye Health 2012;25:41-3.
26. Census Office: The population of towns, villages and associated localities. 2011 Population and Housing Census. Gaborone: Statistics Botswana, 2012.

27. Ntim-Amponsah CT, Winifried MK, Ofosu-Amah S. Awareness and knowledge of glaucoma and other diseases associated with blindness in a Ghanian community. Niger J Ophthalmol 2004;12:50-4.

28. Balo PK, Serouis G, Banla M, et al. Knowledge, attitudes and practices regarding glaucoma in the urban and suburban population of Lomé (Togo). Sante 2004;14:187-91.

29. Bodunde OT, Daneil OJ, Onobolu OO, et al. Knowledge, attitude and health beliefs of glaucoma patients in a Nigerian hospital. Niger Med Pract 2006:50:62-4.

30. Mwanza JC. Primary open-angle glaucoma in sub-Saharan Africa. Niger J Ophthalmol 2006;14:22-6.

31. Adegbehingbe BO, Bisiriyu LA. Knowledge, attitudes, and self care practices associated with glaucoma among hospital workers in Ile-Ife, Osun State, Nigeria. Tanzan J Health Res 2008;10:240-5.

32. Onyekwe LO, Okosa MC, Apakama Al. Knowledge and attitude of eye hospital patients towards chronic open angle glaucoma in Onitsha. Niger Med J 2009;50:1-3.

33. Tenkir A, Solomon B, Deribew A. Glaucoma awareness among people attending ophthalmic outreach services in Southwestern Ethiopia. BMC Ophthalmol 2010;10:17.

34. Nwosu SN. Patients' knowledge of glaucoma and treatment options. Niger J Clin Pract 2010;13:74-7.

35. Abroms LC, Maibach EW. The effectiveness of mass communication to change public behavior. Annu Rev Public Health 2008;29:219-34.

36. Mohamed EA, Bayoumi OR, Draz SF. Impact of an educational programme on knowledge, beliefs, practices and expectations about care among adolescent glaucoma patients in Cairo. East Mediterr Health J 2011;17:960-8.

37. Olawoye O, Fawole OI, Teng CC, et al. Evaluation of community eye outreach programs for early glaucoma detection in Nigeria. Clin Ophthalmol 2013;7:1753-9.

38. Verrey JD, Foster A, Wormald R, et al. Chronic glaucoma in northern Ghana-a retrospective study of 397 patients. Eye 1990;4:115-20.

39. Briesen S, Geneau R, Roberts $\mathrm{H}$, et al. Understanding why patients with cataract refuse free surgery: the influence of rumours in Kenya. Trop Med Int Health 2010;15:534-9.

40. Quigley H, Buhrmann R, West S, et al. Long term results of glaucoma surgery among participants in an east African population survey. Br J Ophthalmol 2000;84:860-4.

41. Melamed S, Ben Simon GL, Levkovitch-Verbin H. Selective laser trabeculoplasty as primary treatment for open-angle glaucoma: a prospective, non-randomized pilot study. Arch Ophthalmol 2003;121:957-60.

42. Abdelrahman AM, Eltanamly RM. Selective laser trabeculoplasty in Egyptian patients with primary open-angle glaucoma. Middle East Afr J Ophthalmol 2012;19:299-303.

43. David $\mathrm{R}$, Stone $\mathrm{D}$. The prevalence of glaucoma in a homogeneous African population. In: Ticho U, David R, eds. Recent advances in glaucoma. New York: Elsevier Science Publishers, 1984:145-53. 